ITEP/TH-9/15

IITP/TH-3/15

\title{
On the defect and stability of differential expansion
}

\author{
Ya.Kononov $^{d}$, A.Morozov ${ }^{a, b, c}$ \\ ${ }^{a}$ ITEP, Moscow 117218, Russia \\ ${ }^{b}$ National Research Nuclear University MEPhI, Moscow 115409, Russia \\ ${ }^{c}$ Institute for Information Transmission Problems, Moscow 127994, Russia \\ ${ }^{d}$ Higher School of Economics, Math Department, Moscow, 117312, Russia
}

\begin{abstract}
Empirical analysis of many colored knot polynomials, made possible by recent computational advances in Chern-Simons theory, reveals their stability: for any given negative $N$ and any given knot the set of coefficients of the polynomial in $r$-th symmetric representation does not change with $r$, if it is large enough. This fact reflects the non-trivial and previously unknown properties of the differential expansion, and it turns out that from this point of view there are universality classes of knots, characterized by a single integer, which we call defect, and which is in fact related to the power of Alexander polynomial.
\end{abstract}

HOMFLY polynomials are Wilson-loop averages in $3 d$ Chern-Simons theory [1], which in this simplest model depend only on the topology of the Wilson line (knot). Therefore one can separate and study the group-theory properties of observables - and this is a non-trivial and very interesting problem, for a brief summary of results see [2]. From the quantum field theory perspective knot polynomials are direct generalization of conformal blocks, and this relation [3] provides one of the effective calculational methods in knot theory.

Recent advances in [4, 5], based on the previous considerations in [3]-[15], provided a way to systematically calculate simplest colored HOMFLY polynomials [16] for a really wide variety of knots - including, in particular, the entire Rolfsen table of [17]. This allows us to return to the study of "differential expansions" of [18]-[27], which was temporarily postponed because of the insufficient "experimental" material.

In this note we describe empirically obtained properties of these expansions for symmetric representations $[r]$ (where $r$ is the length of the single-line Young diagram). It looks like there are different universality classes of such expansions, characterized by a single integer, which we call "defect" $\delta^{\mathcal{K}}$. Moreover, these newly observed properties allow to identify $2\left(\delta^{\mathcal{K}}+1\right)$ with the power of Alexander polynomial and lead to a peculiar stability property of symmetrically colored HOMFLY for large enough $r$ : what stabilizes is not the polynomial itself, but the set of its coefficients - i.e. something like the "coordinates" $g_{r, j}$, introduced in [24]. Theoretical analysis of these observations, proofs and extension to non-(anti)symmetric representations are beyond the scope of the present text.

\section{The notion of defect}

Differential expansion provides a knot-dependent $q$-deformation (quantization) of the remarkable factorization property $[9,11],[19]-[21]$ of colored "special" polynomials at $q=1$,

$$
H_{R}^{\mathcal{K}}(A)=\left.\left(H_{[1]}^{\mathcal{K}}(A)\right)^{|R|}\right|_{q=1} \quad \forall \text { representation } R \text { and knot } \mathcal{K}
$$

which fully defines their dependence on representation (Young diagram) $R$. Currently these expansions can be well studied only for symmetrically-colored HOMFLY, and we focus on this case in the present paper.

The story starts from the fact that

- $H_{r}=H_{[r]}$ always possesses differential expansion of the following form:

$$
H_{r}^{\mathcal{K}}\left(A, q^{2}\right)=1+\sum_{s=1}^{r} \frac{[r] !}{[s] ![r-s] !} \cdot G_{s}^{\mathcal{K}}(A, q) \cdot\{A / q\} \cdot \prod_{j=0}^{s-1}\left\{A q^{r+j}\right\}
$$

For generic knot $G_{s}^{\mathcal{K}}$ is a non-factorizable Laurent polynomial of $A$ and $q$, but for some knots it can be further factorized. In this formula we use the notation $\{x\}=x-x^{-1}$ and quantum number is defined as $[n]=\left\{q^{n}\right\} /\{q\}$.

- What is important, if $G_{s}^{\mathcal{K}}$ is divisible by some "differential" $\left\{A q^{k}\right\}$, the same is true for all other $G_{s^{\prime}}^{\mathcal{K}}$ with $s^{\prime}>s$. This property allows one to introduce defect functions $\nu_{s}^{\mathcal{K}}$ and $\mu_{s}^{\mathcal{K}}=s-1-\nu_{s}^{\mathcal{K}}$ :

$$
G_{s}^{\mathcal{K}}(A, q)=F_{s}^{\mathcal{K}}(A, q) \cdot \prod_{j=0}^{\nu_{s}^{\mathcal{K}}-1}\left\{A q^{j}\right\}=F_{s}^{\mathcal{K}}(A, q) \cdot \prod_{j=0}^{s-2-\mu_{s}^{\mathcal{K}}}\left\{A q^{j}\right\}
$$


which are both(!) monotonically increasing function of $s$,

$$
\nu_{s}^{\mathcal{K}} \leq \nu_{s^{\prime}}^{\mathcal{K}}, \quad \mu_{s}^{\mathcal{K}} \leq \mu_{s^{\prime}}^{\mathcal{K}} \quad \text { for } \quad s<s^{\prime}
$$

i.e. both grow - but not faster than $s$.

- For $A=q^{N}$ with any fixed $N$, positive or negative,

$$
F_{s}\left(q^{N}, q\right) \sim\{q\}^{\mu_{s}^{\mathcal{L}}} \Longleftrightarrow G_{s}\left(q^{N}, q\right) \sim\{q\}^{s-1}
$$

i.e. at fixed $N$ the $s$-the term of differential expansion is actually of the order $\{q\}^{2 s}$.

- It turns out that $\nu_{s}^{\mathcal{K}}$ as a function of $s$ has a very special shape, fully parameterized by a single integer $\delta^{\mathcal{K}} \geq-1$, which we call the defect of differential expansion:

$$
\operatorname{defect} \delta^{\mathcal{K}}=-1 \quad \Longrightarrow \quad \mu_{s}^{\mathcal{K}}=s-2, \quad \nu_{s}^{\mathcal{K}}=1
$$

$$
\operatorname{defect} \delta^{\mathcal{K}}=0 \quad \Longrightarrow \quad \mu_{s}^{\mathcal{K}}=0, \quad \nu_{s}^{\mathcal{K}}=s-1
$$

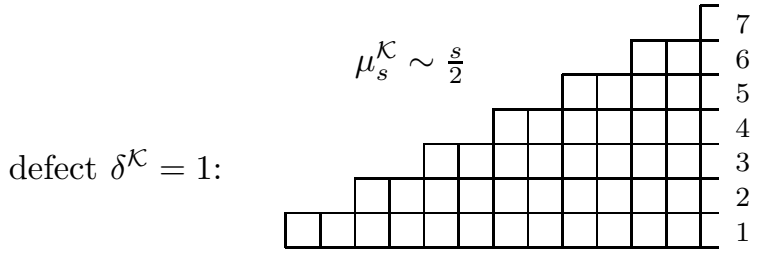

$s$

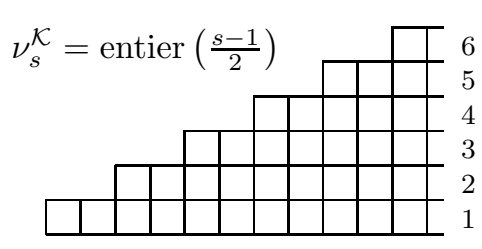

$\begin{array}{lllllllll}s & 3 & 4 & 5 & 6 & 7 & 8 & 9 & 1011121314\end{array}$

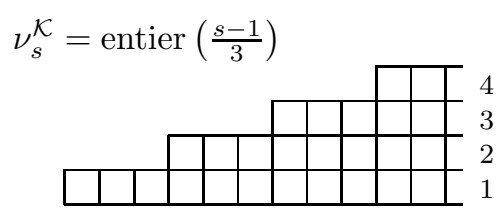

$s \quad 4 \quad 5 \quad 6 \quad 7 \quad 8 \quad 91011121314$

defect $\delta^{\mathcal{K}}=3$ :
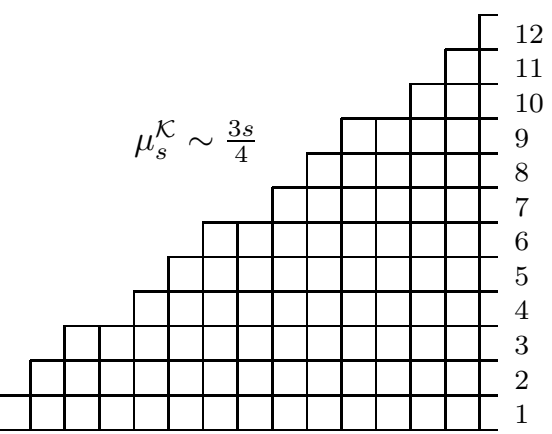

$s \quad 2 \quad 3 \quad 4 \quad 5 \quad 6 \quad 7 \quad 8 \quad 910111213141516$

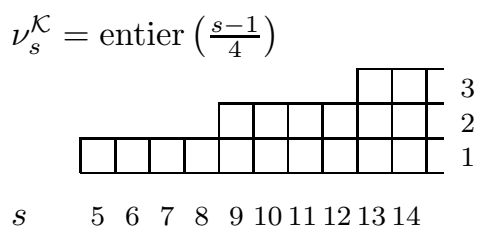

In general

$$
\nu_{s}^{\mathcal{K}}=\operatorname{entier}\left(\frac{s-1}{\delta^{\mathcal{K}}+1}\right) \sim \frac{s}{\delta^{\mathcal{K}}+1}, \quad \quad \mu_{s}^{\mathcal{K}}=s-1-\nu_{s}^{\mathcal{K}} \sim \frac{\delta^{\mathcal{K}}}{\delta^{\mathcal{K}}+1} \cdot s
$$




\section{Relation to Alexander polynomial}

It is an interesting question, if the value of $\delta^{\mathcal{K}}$ can also restrict the coefficient functions $F_{s}^{\mathcal{K}}(A, q)$.

Immediately observable are two remarkable properties of this kind.

- $G_{1}^{\mathcal{K}}(A, q)$ has power $2 \delta^{\mathcal{K}}$ in $q^{2}$, i.e. $G_{1}^{\mathcal{K}}(A, q)=\sum_{j=-\delta^{\mathcal{K}}}^{\delta^{\mathcal{K}}} c_{j} q^{2 j}$.

For example, $\delta^{\mathcal{K}}=0$ whenever $G_{1}^{\mathcal{K}}$ is independent of $q$.

- Alexander polynomial has power $2\left(\delta^{\mathcal{K}}+1\right)$ in $q^{2}$, i.e. $A l^{\mathcal{K}}(q)=H_{1}^{\mathcal{K}}(A=1, q)=\sum_{j=-\delta^{\mathcal{K}}-1}^{\delta^{\mathcal{K}}+1} a_{j} q^{2 j}$

$$
\delta^{\mathcal{K}}=\frac{1}{2} \operatorname{Power}_{q^{2}}\left(A l^{\mathcal{K}}\right)-1
$$

For $\delta^{\mathcal{K}} \neq 0$ these facts are not immediately related: contributing to Alexander polynomials are all $G_{s}^{\mathcal{K}}$ with $s \leq \delta^{\mathcal{K}}+1$ and they can and do contain much higher powers in $q$. Moreover, even the product of differentials in the $s$-term has power in $q$, which grows quadratically with $s$ - and thus with $\delta^{\mathcal{K}}$. This means that there are serious cancelations behind the linear law (12).

Since Alexander polynomials are easily available already from [17], we can now list the defects of all the knots from the Rolfsen table (up to 10 intersections):

\begin{tabular}{c||c|c|cc|ccc|ccccccc|}
$\mathcal{K}$ & $3_{1}$ & $4_{1}$ & $5_{1}$ & $5_{2}$ & $6_{1}$ & $6_{2}$ & $6_{3}$ & $7_{1}$ & $7_{2}$ & $7_{3}$ & $7_{4}$ & $7_{5}$ & $7_{6}$ & $7_{7}$ \\
\hline$\delta^{\mathcal{K}}$ & 0 & 0 & 1 & 0 & 0 & 1 & 1 & 2 & 0 & 1 & 0 & 1 & 1 & 1
\end{tabular}

\begin{tabular}{c||cccccccccc|cccccccccc|c}
$\mathcal{K}$ & $8_{1}$ & $8_{2}$ & $8_{3}$ & $8_{4}$ & $8_{5}$ & $8_{6}$ & $8_{7}$ & $8_{8}$ & $8_{9}$ & $8_{10}$ & $8_{11}$ & $8_{12}$ & $8_{13}$ & $8_{14}$ & $8_{15}$ & $8_{16}$ & $8_{17}$ & $8_{18}$ & $8_{19}$ & $8_{20}$ & $8_{21}$ \\
\hline$\delta^{\mathcal{K}}$ & 0 & 2 & 0 & 1 & 2 & 1 & 2 & 1 & 2 & 2 & 1 & 1 & 1 & 1 & 1 & 2 & 2 & 2 & 2 & 1 & 1
\end{tabular}

\begin{tabular}{c||cccccccccc|cccccccccc|ccccc}
$\mathcal{K}$ & $9_{1}$ & $9_{2}$ & $9_{3}$ & $9_{4}$ & $9_{5}$ & $9_{6}$ & $9_{7}$ & $9_{8}$ & $9_{9}$ & $9_{10}$ & $9_{11}$ & $9_{12}$ & $9_{13}$ & $9_{14}$ & $9_{15}$ & $9_{16}$ & $9_{17}$ & $9_{18}$ & $9_{19}$ & $9_{20}$ & $9_{21}$ & $9_{22}$ & $9_{23}$ & $9_{24}$ & $9_{25}$ \\
\hline$\delta^{\mathcal{K}}$ & 3 & 0 & 2 & 1 & 0 & 2 & 1 & 1 & 2 & 1 & 2 & 1 & 1 & 1 & 1 & 2 & 2 & 1 & 1 & 2 & 1 & 2 & 1 & 2 & 1
\end{tabular} \begin{tabular}{c||ccccc|cccccccccc|ccccccccc}
$\mathcal{K}$ & $9_{26}$ & $9_{27}$ & $9_{28}$ & $9_{29}$ & $9_{30}$ & $9_{31}$ & $9_{32}$ & $9_{33}$ & $9_{34}$ & $9_{35}$ & $9_{36}$ & $9_{37}$ & $9_{38}$ & $9_{39}$ & $9_{40}$ & $9_{41}$ & $9_{42}$ & $9_{43}$ & $9_{44}$ & $9_{45}$ & $9_{46}$ & $9_{47}$ & $9_{48}$ & $9_{49}$ \\
\hline$\delta^{\mathcal{K}}$ & 2 & 2 & 2 & 2 & 2 & 2 & 2 & 2 & 2 & 0 & 2 & 1 & 1 & 1 & 2 & 1 & 1 & 2 & 1 & 1 & 0 & 2 & 1 & 1
\end{tabular}

\begin{tabular}{c||cccccccccc|cccccccccc}
$\mathcal{K}$ & $10_{1}$ & $10_{2}$ & $10_{3}$ & $10_{4}$ & $10_{5}$ & $10_{6}$ & $10_{7}$ & $10_{8}$ & $10_{9}$ & $10_{10}$ & $10_{11}$ & $10_{12}$ & $10_{13}$ & $10_{14}$ & $10_{15}$ & $10_{16}$ & $10_{17}$ & $10_{18}$ & $10_{19}$ & $10_{20}$ \\
\hline$\delta^{\mathcal{K}}$ & 0 & 3 & 0 & 1 & 3 & 2 & 1 & 2 & 3 & 1 & 1 & 2 & 1 & 2 & 2 & 1 & 3 & 1 & 2 & 1
\end{tabular}

\begin{tabular}{c||cccccccccc|cccccccccc}
$\mathcal{K}$ & $10_{21}$ & $10_{22}$ & $10_{23}$ & $10_{24}$ & $10_{25}$ & $10_{26}$ & $10_{27}$ & $10_{28}$ & $10_{29}$ & $10_{30}$ & $10_{31}$ & $10_{32}$ & $10_{33}$ & $10_{34}$ & $10_{35}$ & $10_{36}$ & $10_{37}$ & $10_{38}$ & $10_{39}$ & $10_{40}$ \\
\hline$\delta^{\mathcal{K}}$ & 2 & 2 & 2 & 1 & 2 & 2 & 2 & 1 & 2 & 1 & 1 & 2 & 1 & 1 & 1 & 1 & 1 & 1 & 2 & 2
\end{tabular}

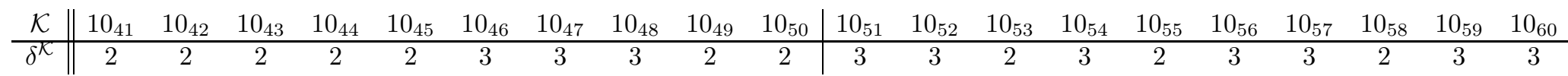

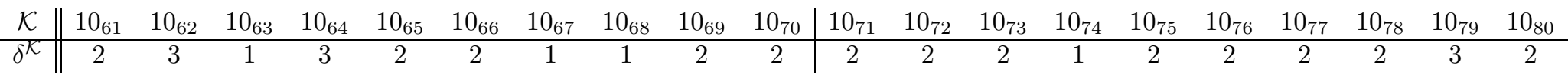

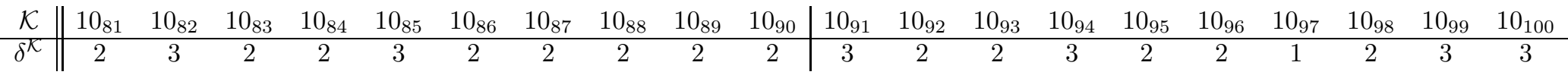
\begin{tabular}{c||cccccccccc|ccccc}
$\mathcal{K}$ & $10_{101}$ & $10_{102}$ & $10_{103}$ & $10_{104}$ & $10_{105}$ & $10_{106}$ & $10_{107}$ & $10_{108}$ & $10_{109}$ & $10_{110}$ & $10_{111}$ & $10_{112}$ & $10_{113}$ & $10_{114}$ & $10_{115}$ \\
\hline$\delta^{\mathcal{K}}$ & 1 & 2 & 2 & 3 & 2 & 3 & 2 & 2 & 3 & 2 & 2 & 3 & 2 & 2 & 2
\end{tabular}

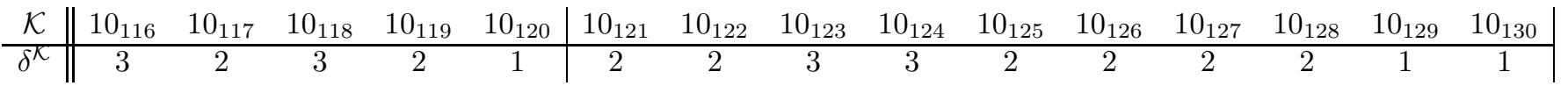
\begin{tabular}{c||cccccccccc|ccccc}
$\mathcal{K}$ & $10_{131}$ & $10_{132}$ & $10_{133}$ & $10_{134}$ & $10_{135}$ & $10_{136}$ & $10_{137}$ & $10_{138}$ & $10_{139}$ & $10_{140}$ & $10_{141}$ & $10_{142}$ & $10_{143}$ & $10_{144}$ & $10_{145}$ \\
\hline$\delta^{\mathcal{K}}$ & 1 & 1 & 1 & 2 & 1 & 1 & 1 & 2 & 3 & 1 & 2 & 2 & 2 & 1 & 1
\end{tabular}

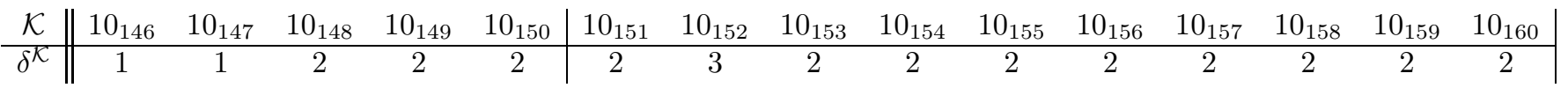
\begin{tabular}{c||ccccc}
$\mathcal{K}$ & $10_{161}$ & $10_{162}$ & $10_{163}$ & $10_{164}$ & $10_{165}$ \\
\hline$\delta^{\mathcal{K}}$ & 2 & 1 & 2 & 1 & 1
\end{tabular} 


\section{Twist and torus knots}

For all twist knots the defect is vanishing

$$
\delta^{\text {twist }}=0
$$

Instead for torus knots it is a kind of maximal:

for the 2-strand family

for the 3 -strand family $\left(8_{19}, 10_{124}, \ldots\right)$

$$
\delta^{[2, n]}=\frac{n-3}{2},
$$

for the 4-strand family $\delta^{[3, n]}=n-2$,

$$
\delta^{[4, n]}=\frac{3 n-5}{2}
$$

$$
\text { in general, } \quad \delta^{[m, n]}=\frac{m n-m-n-1}{2},
$$

since the power of Alexander polynomial $A l^{[m, n]}$ is $(m-1)(n-1)$.

\section{Negative defect: KTC mutants and their relatives}

Starting from 11 intersections there are cases when Alexander is just unity, i.e. the defect is negative, $\delta^{\mathcal{K}}=-1$. According to our general rules this means that for such knots already $G_{1}^{\mathcal{K}}$ is reducible: $G_{1}^{\mathcal{K}} \sim\{A\}$. Of course, also all other $G_{s}^{\mathcal{K}} \sim\{A\}$, because all the terms of the differential expansion are vanishing for $A=1$.

This is indeed true for the first example - the celebrated Kinoshita-Terasaka and Conway $($ KTC) mutants $\mathcal{K}=$ $11 n 42 \& 11 n 34$, reconsidered recently in [5],- and also for the next example, available from [17]: $\mathcal{K}=12 n 313 \& 12 n 430$. Moreover, the combination of [4] and [5] allows to calculate HOMFLY for KTC mutants for any symmetric representation and validate (6) in this particular example.

\section{Summary: stability and other properties of differential expansion}

To explain what we mean by stability, it is simplest to look at an example of a randomly chosen knot $\left(\right.$ say, $\left.\mathcal{K}=6_{2}\right)$ :

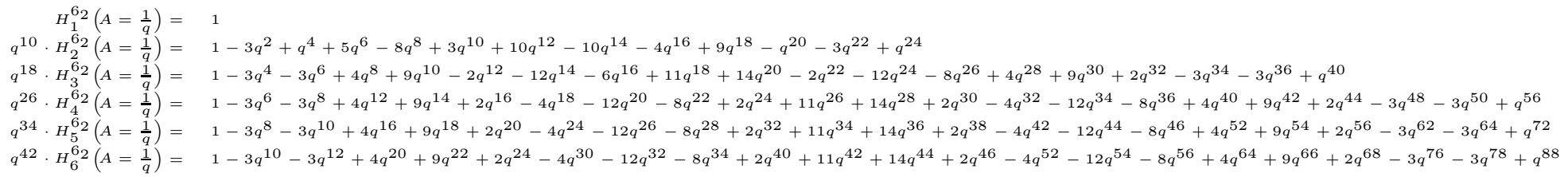

It is easy to observe that, starting from $H_{4}^{6_{2}}$, the sets of coefficients are the same - despite the polynomials are different. At $A=q^{-2}$ the same happens, beginning from $H_{8}^{6_{2}}$. Thus what stabilizes are not the polynomials themselves, but something else, more appropriately associated with the knots. In full accordance with the vision in $[24]$ this something are the coefficient functions $G_{s}^{\mathcal{K}}$ of the differential expansion.

Due to their properties, which are revealed in the present paper, contributing at $A=q^{-N}$ are just the first few terms of the expansion (2):

$$
H_{r}^{\mathcal{K}}\left(A=\frac{1}{q^{N}}\right)=1-\sum_{s=0}^{(N+1)\left(\delta^{\mathcal{K}}+1\right)} \frac{[N+1] \cdot G_{s}^{\mathcal{K}}\left(A=q^{-N}\right)}{\{q\}^{s-1} \cdot[s] !} \prod_{j=0}^{s-1}\left\{q^{r-N+j}\right\}\left\{q^{r-j}\right\}
$$

where the last product is Laurent polynomial in $q^{r}$ and due to (5) the ratio in front of it is an $r$-independent polynomial. Thus what we get is just a sum of a few polynomials, multiplied by different powers of $q^{r}$. They do not overlap at large enough $r$, and this provides an $r$-independent set of the coefficients, as in the above example.

In fact, one could wish to interpret the remarkable identity $[19,20]$

$$
H_{r}^{\mathcal{K}}(A=1, q)=H_{1}^{\mathcal{K}}\left(A=1, q^{r}\right)
$$

for Alexander polynomials as a manifestation of the same phenomenon at $N=0$. However this is literally so only for $\delta^{\mathcal{K}}=-1$ and $\delta^{\mathcal{K}}=0$. Still (16) is true not only for all knots, but actually for all single-hook (and not just single-line) representations $R$. For such representations (16) is a kind of a dual to (1). 


\section{Conclusion}

In this paper we studied the "quality" of the differential expansion (2) for symmetrically colored reduced HOMFLY polynomials - the typical observables in the simplest possible Yang-Mills theory. If only naive representation-theory properties are taken into account from (1) to restriction $l \leq N$ on the number $l$ of lines in the Young diagram for particular $S L(N)$, this expansion has the form (2) with irreducible polynomial coefficient functions $G_{s}(A, q)$. It is well known, however, that sometime $G_{s}$ are further factorized, thus adding more restrictions/structures to the colordependence of physical observables. Now, when methods were developed to study entire classes of generic knots, we could attack this problem in a systematic way - and demonstrate that $G_{s}$ are always factorizable for high enough $s$. The depth of factorization appeared to depend on a single characteristic of the knot, which we originally called defect of the expansion, and further demonstrated that it is linearly related to the degree of Alexander polynomial, what makes it very easy to find.

This factorization universality leads to remarkable kind of stabilization of symmetrically colored HOMFLY - ensuring that increasing $r$ beyond some knot-dependent boundary does not provide new physical (topological) information. This is what one naturally expects, and now we see how this actually works.

Highly desirable is extension of this new insight beyond pure symmetric and antisymmetric representations, but this requires further development of technical tools in conformal, quantum group and $\mathcal{R}$-matrix theories.

\section{Acknowledgements}

Our work is partly supported by grants NSh-1500.2014.2, RFBR 13-02-00478 and by the joint grants 15-52-50041-YaF, 14-01-92691-Ind-a, 15-51-52031-NSC-a. Acknowledged is also support by Brazilian Ministry of Science, Technology and Innovation through the National Counsel of Scientific and Technological Development and by Laboratories of Algebraic Geometry and Mathematical Physics, HSE.

\section{References}

[1] S.-S.Chern and J.Simons, Ann.Math. 99 (1974) 48-69

[2] A.Mironov and A.Morozov, AIP Conf.Proc. 1483 (2012) 189-211, arXiv:1208.2282

[3] E.Witten, Comm.Math.Phys. 121 (1989) 351

[4] D.Galakhov, D.Melnikov, A.Mironov, A.Morozov and A.Sleptsov, Phys.Lett. B743 (2015) 71-74, arXiv:1412.2616; arXiv:1412.8432; arXiv:1502.02621

[5] A.Mironov, A.Morozov, And.Morozov, P.Ramadevi and Vivek Kumar Singh, arXiv:1504.00371

[6] N.Yu.Reshetikhin and V.G.Turaev, Comm. Math. Phys. 127 (1990) 1-26

E.Guadagnini, M.Martellini and M.Mintchev, Clausthal 1989, Procs.307-317; Phys.Lett. B235 (1990) 275

V. G. Turaev and O. Y. Viro, Topology 31, 865 (1992)

A.Morozov and A.Smirnov, Nucl.Phys. B835 (2010) 284-313, arXiv:1001.2003

A.Smirnov, Proc. of International School of Subnuclar Phys. Erice, Italy, 2009, arXiv:hep-th/0910.5011

[7] R.K.Kaul, T.R.Govindarajan, Nucl.Phys. B380 (1992) 293-336, hep-th/9111063; ibid. B393 (1993) $392-412$

P.Ramadevi, T.R.Govindarajan and R.K.Kaul, Nucl.Phys. B402 (1993) 548-566, hep-th/9212110; Mod.Phys.Lett. A9 (1994) 3205-3218, hep-th/9401095; Nucl.Phys. B422 (1994) 291-306, hep-th/9312215; Mod.Phys.Lett. A10 (1995) 1635-1658, hep-th/9412084;

P.Ramadevi and T.Sarkar, Nucl.Phys. B600 (2001) 487-511, hep-th/0009188

[8] R.Gopakumar and C.Vafa, Adv.Theor.Math.Phys. 3 (1999) 1415-1443, hep-th/9811131

H.Ooguri and C.Vafa, Nucl.Phys. B577 (2000) 419-438, arXiv:hep-th/9912123

M.Khovanov, Duke Math.J. 101 (2000) no.3, 359426, math/9908171

S.Gukov, A.Schwarz and C.Vafa, Lett.Math.Phys. 74 (2005) 53-74, hep-th/0412243

E.Witten, arXiv:1401.6996

[9] K. Liu and P. Peng, arXiv:0704.1526; arXiv:1012.2636

[10] M.Aganagic and Sh.Shakirov, arXiv:1105.5117; arXiv:1202.2489; arXiv:1210.2733

S.Arthamonov and Sh.Shakirov, arXiv:1504.02620

[11] P. Dunin-Barkowski, A. Mironov, A. Morozov, A. Sleptsov and A. Smirnov, JHEP 03 (2013) 021, arXiv:1106.4305 
[12] I.Cherednik, arXiv:1111.6195

I.Cherednik and I.Danilenko, arXiv:1408.4348

[13] A.Mironov, A.Morozov and And.Morozov, arXiv:1112.5754; JHEP 03 (2012) 034, arXiv:1112.2654

H. Itoyama, A. Mironov, A. Morozov and And. Morozov, IJMP A27 (2012) 1250099, arXiv:1204.4785 A28 (2013) 1340009, arXiv:1209.6304

A.Anokhina, A.Mironov, A.Morozov and And.Morozov, Adv.High Energy Physics, 2013 (2013) 931830, arXiv: 1304.1486

A.Anokhina and And.Morozov, Theor.Math.Phys. 178 (2014) 1-58, arXiv:1307.2216;

A.Anokhina, arXiv:1412.8444

[14] P.Ramadevi and Zodinmawia, arXiv:1107.3918; arXiv:1209.1346

S.Nawata, P.Ramadevi, Zodinmawia and X.Sun, JHEP 1211 (2012) 157, arXiv:1209.1409

S.Nawata, P.Ramadevi and Zodinmawia, J.Knot Theory and Its Ramifications 22 (2013) 13, arXiv:1302.5144 JHEP 1401 (2014) 126, arXiv:1310.2240

Zodinmawia's PhD thesis, 2014

S.Nawata, P.Ramadevi and Vivek Kumar Singh, arXiv:1504.00364

[15] J.Gu and H.Jockers, arXiv:1407.5643

[16] J.W.Alexander, Trans.Amer.Math.Soc. 30 (2) (1928) 275-306

J.H.Conway, Algebraic Properties, In: John Leech (ed.), Computational Problems in Abstract Algebra, Proc. Conf. Oxford, 1967, Pergamon Press, Oxford-New York, 329-358, 1970

V.F.R.Jones, Invent.Math. 72 (1983) 1; Bull.AMS 12 (1985) 103; Ann.Math. 126 (1987) 335

L.Kauffman,Topology 26 (1987) 395

P.Freyd, D.Yetter, J.Hoste, W.B.R.Lickorish, K.Millet, A.Ocneanu, Bull. AMS. 12 (1985) 239

J.H.Przytycki and K.P.Traczyk, Kobe J. Math. 4 (1987) 115-139

[17] D.Bar-Natan, http://www.katlas.org

[18] N.M.Dunfield, S.Gukov and J.Rasmussen, Experimental Math. 15 (2006) 129-159, math/0505662

[19] H.Itoyama, A.Mironov, A.Morozov and And.Morozov, JHEP 2012 (2012) 131, arXiv:1203.5978

[20] Shengmao Zhu, JHEP 10 (2013) 1-24, arXiv:1206.5886

[21] Anton Morozov, JHEP 1212 (2012) 116, arXiv:1208.3544; JETP Lett. 97 (2013) 171-172, arXiv:1211.4596

[22] A.Mironov, A.Morozov and And.Morozov, AIP Conf. Proc. 1562 (2013) 123, arXiv:1306.3197

[23] E.Gorsky, S.Gukov and M.Stosic, arXiv:1304.3481

[24] S.Arthamonov, A.Mironov and A.Morozov, Theor.Math.Phys. 179 (2014) 509-542, arXiv:1306.5682

[25] S.Arthamonov, A.Mironov, A.Morozov and And.Morozov, JHEP 04 (2014) 156, arXiv:1309.7984

[26] A.Anokhina, A.Mironov, A.Morozov and And.Morozov, Nucl.Phys. B 882C (2014) 171-194, arXiv:1211.6375; Mod.Phys.Lett. A29 (2014) 1450183, arXiv:1408.3076

[27] A.Mironov, A.Morozov and A.Sleptsov, Theor.Math.Phys. 177 (2013) 1435-1470, arXiv:1303.1015; Eur.Phys.J. C73 (2013) 2492, arXiv:1304.7499

A.Mironov, A.Morozov, A.Sleptsov and A.Smirnov, Nucl.Phys. B889 (2014) 757-777, arXiv:1310.7622 